Recepción: 20 / 04 / 2017

Aceptación: 20 / 05 / 2017

Publicación: 15 / 06 / 2017

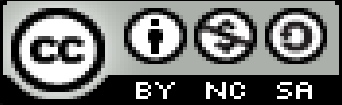

Artículo de Investigación

\title{
Determinación del coeficiente de descarga de un prototipo tubo de Venturi construido para uso académico en laboratorio
}

\section{Determination of the coefficient of discharge of a prototype venturi tube built for academic use in laboratory}

\section{Determinação do coeficiente de descarga de um protótipo de tubo venturi construído para uso acadêmico em laboratorio}

\author{
Jaime A. Pazmiño-Mayorga ${ }^{\mathrm{I}}$ \\ jpazminom@uce.edu.ec \\ Ramiro J. Vivas-Vivas II \\ rjvivas@uce.edu.ec
Valdano L. Tafur-Recalde ${ }^{\text {III }}$ vtafur@uce.edu.ec \\ Wilman I. Ordóñez-Pizarro ${ }^{\text {IV }}$ \\ wiordonez@uce.edu.ec
}

\section{Correspondencia: jpazminom@uce.edu.ec}

\footnotetext{
I. Magister en Docencia Universitaria y Administración Educativa; Ingeniero Civil; Profesor de la Facultad Ciencias Agrícolas de la Universidad Central del Ecuador, Quito, Ecuador.

II. Magister en Educación Superior; Licenciado en Ciencias de la Educación Profesor de Enseñanza media en Educación Técnica Especialidad Electricidad; Profesor de la Facultad Ciencias Agrícolas de la Universidad Central del Ecuador, Quito, Ecuador.

III. Magister en Economía Agrícola y Desarrollo Sustentable; Especialista en Floricultura; Ingeniero Agrónomo; Profesor de la Facultad Ciencias Agrícolas de la Universidad Central del Ecuador, Quito, Ecuador.

IV. Magister en Desarrollo Educativo; Doctor en Psicología Educativa y Orientación Especialización Psicología Infantil; Licenciado en Ciencias de la Educación Profesor de Enseñanza media en la Especialización de Psicología Educativa y Orientación; Profesor de la Facultad de Filosofía, Letras y Ciencias de la Educación de la Universidad Central del Ecuador, Quito, Ecuador.
} 


\title{
Resumen
}

En la Unidad de Física de la Universidad Central del Ecuador en el estudio de la hidrodinámica se requiere determinar el coeficiente de descarga de un modelo de tubo de Venturi diseñado y construido para uso experimental en laboratorio; inicialmente este sistema permite tener régimen laminar y caudal constante, lo que posibilita la determinación del caudal práctico y al poder aplicar la Ecuación de Bernoulli se puede determinar el caudal teórico, al comparar estos dos caudales se determina el coeficiente de descarga.

Palabras claves: Fundamentos de flujo en tuberías; frasco de Mariotte; ecuación de Bernoulli; medidores de Caudal; tubo de Venturi; coeficiente de descarga.

\begin{abstract}
In the Unit of Physics of the Central University of Ecuador in the study of hydrodynamics it is necessary to determine the discharge coefficient of a Venturi tube model designed and constructed for experimental use in the laboratory; Initially this system allows to have laminar regime and constant flow, which makes possible the determination of the practical flow and to be able to apply the Bernoulli equation can determine the theoretical flow rate, when comparing these two flows the discharge coefficient is determined.
\end{abstract}

Key words: Basics of piping flow; bottle of mariotte; bernoulli Equation; flow meters; venturi Tube; coefficient of discharge.

\section{Resumo}

Na Unidade de Física da Universidade Central do Equador no estudo da hidrodinâmica, é necessário determinar o coeficiente de descarga de um modelo de tubo de Venturi projetado e construído para uso experimental no laboratório; Inicialmente, este sistema permite ter regime laminar e fluxo constante, o que possibilita a determinação do fluxo prático e para poder aplicar a equação de Bernoulli, pode determinar o caudal teórico, ao comparar esses dois fluxos, o coeficiente de descarga é determinado.

Palavras-chave: Fundamentos do fluxo de tubulação; Garrafa de mariotte; Equação bernoulli; Medidores de fluxo; Tube venturi; Coeficiente de descarga. 


\section{Introducción.}

En la Unidad de Física de la Universidad Central del Ecuador se diseñó y construyó un modelo de medidor de Venturi con fines didácticos, los autores Pazmiño, García, Fernández y Cisneros demostraron mediante investigación experimental de laboratorio el correcto funcionamiento de este sistema. La presente investigación se plantea determinará experimentalmente en laboratorio el coeficiente de descarga del modelo de medidor de Venturi mencionado, por lo que se debe medir y calcular el caudal práctico y teórico.

El fundamento teórico que permite fundamentar esta investigación es la siguiente:

- Fundamentos de flujo en tuberías

- Frasco de Mariotte

- Ecuación de Bernoulli

- Medidores de caudal

- Tubo de Venturi

- Coeficiente de descarga

\section{Fundamentos del flujo en tuberías}

\section{Número de Reynolds}

Este parámetro adimensional es una medida de la razón de la fuerza de inercia sobre un elemento del fluido a la fuerza viscosa sobre un elemento. (Martínez E., Navarrete C. 2000.)

El número de Reynolds está definido por la siguiente ecuación:

$\rho=$ densidad del fluido

$\mathrm{V}=$ velocidad característica del fluido 
$\mathrm{D}=$ diámetro de la tubería a través de la cual circula el fluido

= viscosidad dinámica del fluido

\section{Flujo laminar}

En el flujo laminar las partículas del fluido solo se mezclan a escala molecular, de modo que, durante el movimiento, dichas partículas se desplazan según trayectorias paralelas bajo la acción de la viscosidad. En la práctica, el flujo laminar se produce cuando el número de Reynolds no excede los valores de 1.500 a 2.000. (Martínez E., Navarrete C. 2000)

\section{Flujo turbulento}

En el flujo turbulento las partículas del fluido se mezclan a escala molar, de modo que durante el movimiento se produce un intercambio de cantidad de movimiento entre partículas adyacentes, ocasionando una rápida y continua agitación y mezcla en el seno del fluido. En la práctica el flujo turbulento se produce para números de Reynolds por encima de valores entre 6.000 a 10.000. (Martínez E., Navarrete C. 2000)

\section{Pérdida de energía}

También es llamada pérdida de carga y es la pérdida de energía que experimentan los líquidos que fluyen en tuberías y canales abiertos. La energía necesaria para vencer los efectos del rozamiento en el flujo turbulento es la pérdida de carga. Las pérdidas de energía localizadas en las turbulencias incluidas por las piezas especiales y los accesorios que se utilizan en tuberías y canales son también pérdidas de carga. La pérdida de carga se representa habitualmente por el símbolo hL. (Martínez E., Navarrete C. 2000) 


\section{Línea piezométrica}

Línea piezométrica como muestra el Gráfico No. 1, es la línea que une los puntos hasta los que el líquido podría ascender si se insertan tubos piezométricos en distintos lugares a lo largo de la tubería o canal abierto. Es una medida de la altura de presión hidrostática disponible en dichos puntos. (Martínez E., Navarrete C. 2000)

\section{Línea de energía}

También es llamada línea de carga. La energía total del flujo en cualquier sección, con respecto a un plano de referencia determinado, es la suma de la altura geométrica o de elevación Z, la altura piezométrica o de carga, y, y la altura cinética o de presión dinámica $\mathrm{V}^{2} / 2 \mathrm{~g}$. La variación de la energía total de una sección a otra se representa por una línea denominada de carga o de energía y también gradiente de energía, ver Gráfico 1. En ausencia de pérdidas de energía, la línea de carga se mantendrá horizontal, aun cuando podría variar la distribución relativa de la energía entre las alturas geométrica, piezométrica y cinética. Sin embargo, en todos los casos reales se producen pérdidas de energía por rozamiento y la línea de carga resultante es inclinada. (Martínez E., Navarrete C. 2000)

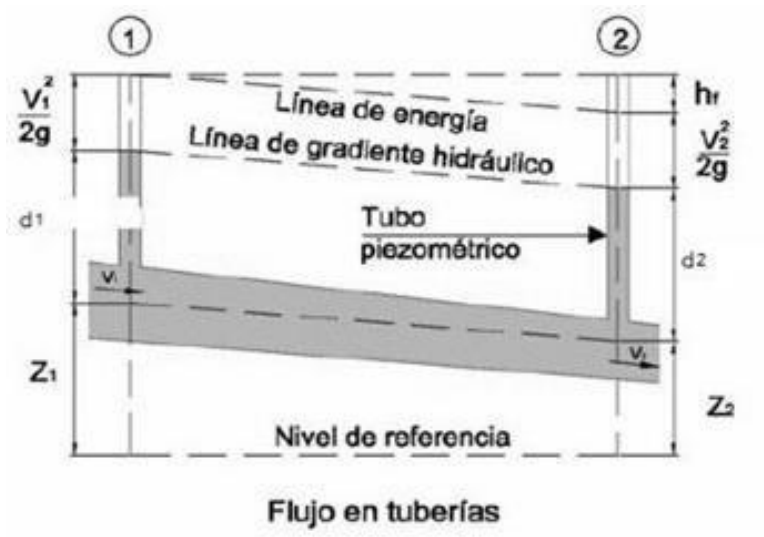

Gráfico $N^{\circ}$ 1.- Línea de Energía o Gradiente de Energía 


\section{Flujo permanente}

El flujo permanente se produce cuando la descarga o caudal en cualquier sección transversal permanece constante. (Martínez E., Navarrete C. 2000.)

\section{Flujo uniforme y no uniforme}

Se llama flujo uniforme aquel en que el calado, sección transversal y demás elementos del flujo se mantienen sustancialmente constantes de una sección a otra. Si la pendiente sección transversal y velocidad cambian de un punto a otro de la conducción, el flujo se dice no uniforme. Un ejemplo de flujo permanente no uniforme es aquel que atraviesa un tubo Venturi utilizado para medir caudales. (Martínez E., Navarrete C. 2000.)

\section{Frasco de Mariotte}

El Frasco de Mariotte es un artefacto destinado a conseguir una velocidad constante de salida de un líquido, el mismo se describe en el Figura 1. Para la utilización del mismo se debe considerar que entre el recipiente y la tubería vertical se produce un cierre hermético, que esta tubería y el orificio de salida son de pequeño diámetro y también que la velocidad de salida es función directa de la altura $(\mathrm{H})$ de líquido considerada entre el extremo inferior de la tubería y el centro del orificio. Es necesario acoplar el frasco de Mariotte al sistema para la determinar de las pérdidas de energía por rozamiento, ya que el mismo nos permite tener un caudal constante. (Pazmiño, García, Fernández, Cisneros, 2017) 


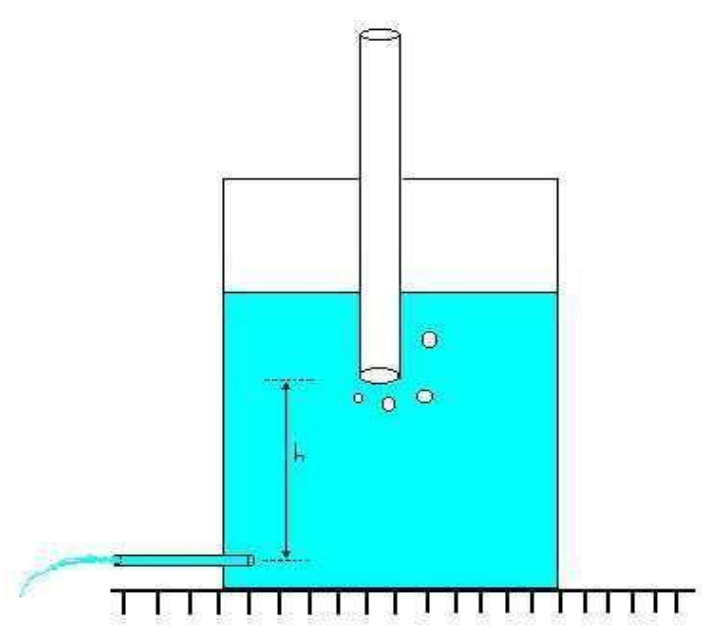

Figura $N^{\circ}$ 1.- Frasco de Mariotte

\section{Ecuación de Bernoulli}

La hidrodinámica es una de las ramas de la Física que más interacción tiene con la ingeniería y con los fenómenos de la naturaleza. Estudia las propiedades de los fluidos hídricos, las principales leyes de la mecánica aplicadas a los mismos y las diferentes teorías que soportan el conocimiento de esta importante rama de la ciencia. En la Universidad Central del Ecuador se estudia hidrodinámica como parte de las mallas curriculares. (Pazmiño et al., 2017)

El principio de Bernoulli, también denominado ecuación de Bernoulli o Trinomio de Bernoulli, describe el movimiento de un fluido a lo largo de un tubo de corriente. Fue expuesto por Daniel Bernoulli en su obra Hidrodinámica (1738) y expresa que: “La energía total por unidad de volumen de un fluido incompresible animado de movimiento estacionario es constante" (fluido ideal). (Serway, Raymond A. 2008)

Según la investigación realizado por (Pazmiño et al., 2017), la ecuación de Bernoulli consta de los siguientes elementos: 
Energía Cinética por unidad de volumen: es la energía debida a la velocidad que posea el fluido.

Conocida como presión dinámica.

\section{Ecuación $N^{\circ}$ 1.- Energía Cinética por unidad de volumen}

Energía Potencial Gravitacional por unidad de volumen: es la energía debido a la altitud que un fluido posee. Conocida como presión hidráulica o altimétrica.

\section{Ecuación $N^{\circ}$ 2.- Energía Potencial Gravitacional por unidad de volumen}

Energía de flujo o Energía Potencial de Presión por unidad de Volumen: es la energía que un fluido contiene debido a la presión que posee. Conocida como presión estática.

La suma de estas energías se conoce como "Ecuación de Bernoulli” (Trinomio de Bernoulli)

Ecuación $N^{\circ}$ 3.- Ecuación de Bernoulli o Conservación de la energía del fluido 
En donde:

Velocidad del fluido en la sección considerada.

Densidad del fluido.

Presión a lo largo de la línea de corriente.

Aceleración gravitatoria.

Altura en la dirección de la gravedad desde una cota de referencia.

La Ecuación 3 representa la conservación de la energía del fluido usada en fluidos ideales y su uso es limitado para fluidos compresibles como los gases.

En el movimiento de un fluido líquido por una tubería de sección variable se deduce que en los lugares de menor área de la tubería aumente la velocidad del fluido, disminuyendo la presión estática, caso contrario en los lugares de mayor área.

En esta investigación es fundamental que el prototipo utilizado cumpla con las condiciones de utilización de la Ecuación de Bernoulli y poder medir adecuadamente la pérdida de energía por rozamiento.

\section{Medidores de Caudal}

Es un dispositivo que instalado en una tubería permite determinar el caudal que circula por la misma. La mayor parte de medidores de caudal son construidos reduciendo el área de flujo, por lo que se determina que en el estrechamiento se gana energía de velocidad, pero se pierde energía de presión, posteriormente con los datos obtenidos se determina el caudal aplican la ecuación de Bernoulli. Uno de los tipos de medidor de caudal más utilizado es el Tubo de Venturi. 


\section{Tubo de Venturi}

\section{Descripción de tubo de Venturi}

El tubo de Venturi se utiliza para medir la velocidad de un fluido incompresible. Consiste en un tubo con un estrechamiento, de modo que las secciones antes y después del estrechamiento son $A_{1}$ y $A_{2}$, con $A_{1}>A_{2}$. En cada parte del tubo hay un manómetro, de modo que se pueden medir las presiones respectivas $\mathrm{p}_{1}$ y $\mathrm{p}_{2}$. Encuentra una expresión para la velocidad del fluido en cada parte del tubo en función del área de las secciones, las presiones y su densidad. (Serway, Raymond A. 2008)

En el Figura 2 siguiente se observa el esquema elemental del tubo de Venturi utilizado.

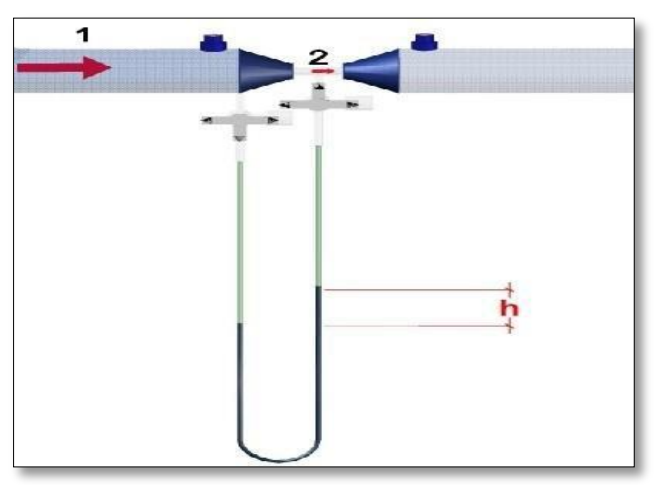

Figura $N^{\circ}$ 2.- Esquema elemental de tubo Venturi horizontal

A continuación, se indica el tubo de Venturi utilizado, el cual fue diseñado y desarrollado por Pazmiño, García, Fernández y Cisneros en su artículo "Diseño y construcción de un prototipo de medidor de Venturi con fines didácticos y aplicación a la ingeniería agronómica”, en el 2017. Ver Figura 3 y Figura 4. 

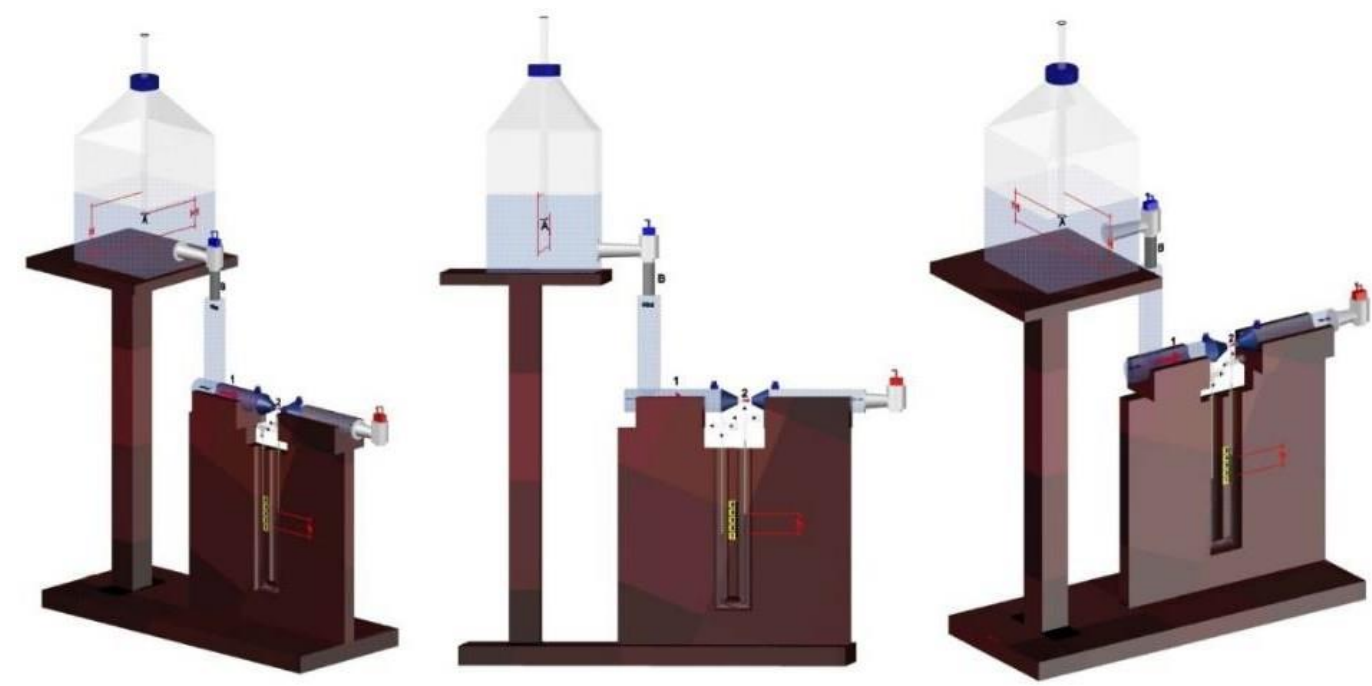

Figura $N^{\circ}$ 3.- Esquema de Sistema de medidor de Venturi utilizado en esta investigación

\section{Determinación de velocidad y caudal mediante tubo de Venturi}

A continuación, en Gráfico 2 la representación de variables para la determinación de velocidad y caudal en el tubo de Venturi.

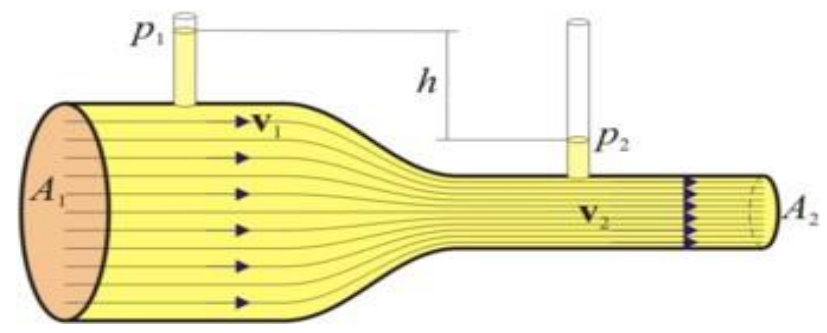

\section{Gráfico $N^{\circ}$ 2.- Representación gráfica de variables en el tubo de Venturi}

La ley de conservación de la masa establece que en un flujo estacionario toda la masa que entra por un lado de un recinto debe salir por otro, lo que implica que la velocidad debe ser mayor en la parte más estrecha del tubo, entonces: 


$$
v_{1} A_{1}=v_{2} A_{2}
$$

Por otro lado, la ley de Bernoulli establece que para dos puntos situados en la misma línea de corriente se cumple:

$$
p_{1}+\rho g y_{1}+\frac{1}{2} \rho v_{1}^{2}=p_{2}+\rho g y_{2}+\frac{1}{2} \rho v_{2}^{2}
$$

Si los dos puntos se encuentran a la misma altura la presión hidrostática es la misma para ambos, por lo que:

$$
p_{1}+\frac{1}{2} \rho v_{1}^{2}=p_{2}+\frac{1}{2} \rho v_{2}^{2}
$$

Reordenando términos, entonces:

$$
v_{1}^{2}-v_{2}^{2}=\frac{2\left(p_{2}-p_{1}\right)}{\rho}
$$

Sustituimos la ecuación de conservación de la masa, entonces:

$$
v_{1}^{2}\left(1-\frac{A_{1}^{2}}{A_{2}^{2}}\right)=\frac{2\left(p_{2}-p_{1}\right)}{\rho} \Rightarrow v_{1}=A_{2} \sqrt{\frac{2\left(p_{2}-p_{1}\right)}{\rho\left(A_{1}^{2}-A_{2}^{2}\right)}}
$$

Análogamente,

$$
v_{2}=A_{1} \sqrt{\frac{2\left(p_{2}-p_{1}\right)}{\rho\left(A_{1}^{2}-A_{2}^{2}\right)}}
$$

y el flujo volumétrico es:

$$
Q=A_{1} v_{1}=A_{2} v_{2}=A_{1} A_{2} \sqrt{\frac{2\left(p_{2}-p_{1}\right)}{\rho\left(A_{1}^{2}-A_{2}^{2}\right)}}
$$


Si la diferencia de presiones se mide a partir de la diferencia de altura en dos manómetros, esta ecuación queda de la siguiente manera:

$$
Q=A_{1} v_{1}=A_{2} v_{2}=A_{1} A_{2} \sqrt{\frac{2 g h}{\rho\left(A_{1}^{2}-A_{2}^{2}\right)}}
$$

\section{Coeficiente de descarga}

El caudal teórico calculado a partir de la Ecuación de Bernoulli (Q Teórico) no es igual al caudal real obtenido en forma práctica ( $Q$ Real), ya que no considera las pérdidas de energía existentes en el sistema de Tubo de Venturi utilizado. Para poder obtener el caudal real se debe multiplicar al caudal teórico por lo el coeficiente de descarga $\left(\mathrm{C}_{\mathrm{d}}\right)$, la ecuación que considera lo mencionado se indica a continuación.

$$
\mathrm{Q}_{\text {real }}=\mathrm{C}_{\mathrm{d}} * \mathrm{Q} \text { teórico }
$$

Una vez obtenido el caudal real se pueden obtener las velocidades reales de flujo, dividiendo el mismo para la correspondiente área de la sección transversal.

En la presente investigación se determinará experimentalmente en el laboratorio el valor del Caudal Teórico y Caudal Práctico para la determinación del coeficiente de descarga $\mathrm{C}_{\mathrm{d}}$.

\section{Metodología.}

El enfoque de esta investigación es el análisis de los datos obtenidos de tipo cuantitativo y cualitativo, al asumir en el experimento la cuantificación de los datos y a su vez la descripción de su comportamiento tomando en consideración los factores estudiados. En este caso las variables son: el 
flujo del agua dentro del tubo de Venturi y las correspondientes pérdidas de energía que tienen correspondencia con el caudal teórico y caudal práctico. Al ser un experimento de laboratorio realizado por estudiantes con asistencia del profesor esta investigación es empírica-analítica. Por tener como propósito la comprobación de una pregunta de investigación por medio de modelos cuantitativos esta investigación se ubica en el área de las ciencias exactas. Al manipular deliberadamente las variables vinculadas a las causas con la finalidad de medir el efecto en la variable de interés esta investigación es de tipo experimental (Sampieri, 2014). Estos datos obtenidos experimentalmente en el Laboratorio de la Unidad de Física de la Universidad Central del Ecuador, fueron obtenidos utilizando el modelo de tubo de Venturi que fue diseñado y construido por Pazmiño, et al (2017).

\section{Pregunta de Investigación:}

¿Existe un procedimiento experimental de laboratorio y su correspondiente modelo físicomatemático que permita utilizando un modelo de tubo de Venturi determinar el coeficiente de descarga producido por pérdidas de energía?

Las constantes y variables utilizadas en esta investigación son:

Aceleración de la gravedad

Densidad del agua

Densidad del mercurio

Volumen

Tiempo

Caudal Práctico

Diferencia de altura de mercurio en el tubo de Venturi (Diferencia de Presión) 
Posición y Velocidad de la partícula de líquido en la sección transversal mayor y menor

Caudal Teórico

\section{Resultados y discusión}

Determinación del coeficiente de descarga con datos experimentales obtenidos del modelo de tubo de Venturi

A) Determinación del número de Reynolds

\begin{tabular}{c|c|c|c|c|c|c}
\hline $\begin{array}{c}\text { Sección } \\
\text { Transv. }\end{array}$ & $\begin{array}{c}\text { Densidad } \\
(\mathrm{H} 2 \mathrm{O}) \mathrm{Kg} / \mathrm{m}^{3}\end{array}$ & $\begin{array}{c}\text { Diámetro } \\
\mathrm{m}\end{array}$ & $\begin{array}{c}\text { Área } \\
\mathrm{m}^{2}\end{array}$ & $\begin{array}{c}\text { Velocidad } \\
\mathrm{m} / \mathrm{s}\end{array}$ & $\begin{array}{c}\text { Viscosidad } \\
\text { Cinemática } \\
\left(\mathrm{H} 2 \mathrm{O}-15{ }^{\circ} \mathrm{C}\right)\end{array}$ & $\begin{array}{c}\text { Número de } \\
\text { Reynols }\end{array}$ \\
\hline mayor & 1000 & 0,02947 & $6,82 \mathrm{E}-04$ & 0,01363 & 0,00139 & 289,0 \\
\hline menor & 1000 & 0,00823 & $5,32 \mathrm{E}-05$ & 0,17440 & 0,00139 & 1032,6 \\
\hline
\end{tabular}

Tabla $N^{\circ}$ 1.- Determinación del Número de Reynols

Del resultado obtenido se desprende que el flujo de agua se encuentra en régimen laminar, porque el valor del número de Reynolds es menor a 2100, también el flujo se mantiene estacionario y se comporta como si estuviera formado por láminas delgadas, que interactúan sólo en función de los esfuerzos tangenciales existentes. 


\section{B) Determinación del Caudal Práctico Promedio}

\begin{tabular}{c|c|c|c|c|c|c|c|c}
\hline \multirow{2}{*}{ Volumen } & $\begin{array}{c}\mathrm{T}_{1} \\
(\mathrm{~S})\end{array}$ & $\begin{array}{c}\mathrm{T} 2 \\
(\mathrm{~S})\end{array}$ & $\begin{array}{c}\mathrm{T} 3 \\
(\mathrm{~S})\end{array}$ & $\begin{array}{c}\mathrm{T} 4 \\
(\mathrm{~S})\end{array}$ & $\begin{array}{c}\mathrm{T}_{5} \\
(\mathrm{~S})\end{array}$ & $\begin{array}{c}\text { T Promedio } \\
(\mathrm{S})\end{array}$ & $\begin{array}{c}\text { Q Práctico } \\
\left(\mathrm{m}^{3} / \mathrm{s}\right) * \\
10^{-6}\end{array}$ & $\begin{array}{c}\text { Q Práctico } \\
\text { Promedio } \\
\left(\mathrm{m}^{3} / \mathrm{s}\right) * \\
10^{-6}\end{array}$ \\
\hline 100 & 2,84 & 2,48 & 3,02 & 3,23 & 2,85 & 2,88 & 34,67 & \\
\hline 200 & 5,79 & 5,15 & 5,68 & 6,34 & 6,08 & 5,81 & 34,44 & 34,24 \\
\hline 300 & 8,75 & 8,39 & 8,68 & 9,4 & 9,39 & 8,92 & 33,62 & \\
\hline
\end{tabular}

Tabla $N^{\circ}$ 2.- Determinación del Caudal Práctico Promedio

C) Solución al modelo físico-matemático que determina el coeficiente de descarga en el sistema de tubo de Venturi utilizado

$\underline{\text { Datos }}$

Número de Reynolds máximo = 1032,6

Q Práctico Promedio $=34,24 * 10^{-6} \mathrm{~m}^{3} / \mathrm{s}$

$\lfloor$ †

士

$[-]$

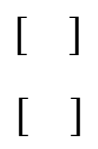

[]

Pol. Con. (Edición núm. 7) Vol. 2, No 6, junio 2017, pp. 1081-1102, ISSN: 2550 - 682X 


\section{Gráfico}

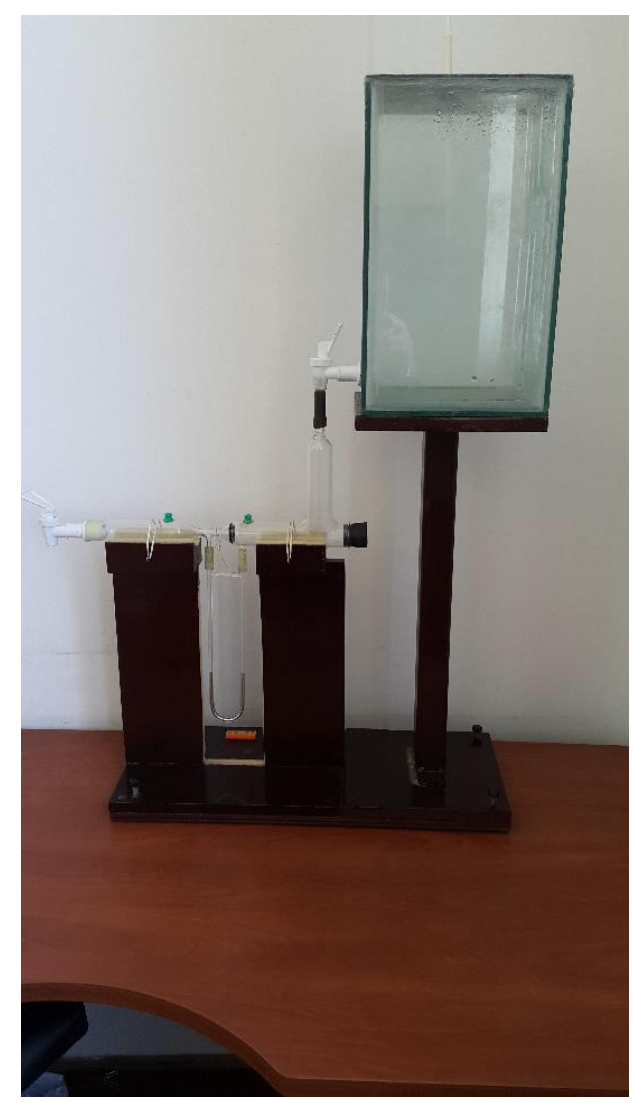

Figura $N^{\circ}$ 4.- Modelo de tubo de Venturi utilizado

\section{Modelo Físico-Matemático y Solución}

Determinación de la diferencia de presión, entre la sección transversal mayor y sección transversal menor del tubo de Venturi:

Determinación de la diferencia de presión $\left(\mathrm{P}_{1}-\mathrm{P}_{2}\right)$ :

() 
( )

()

$(+$

Aplicación de la Ecuación de Bernoulli:

()()

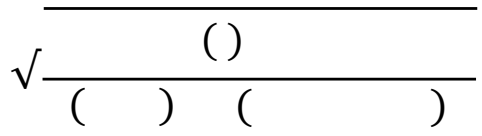

(

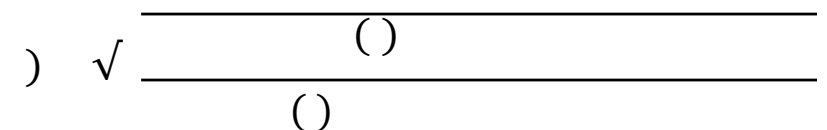

( )

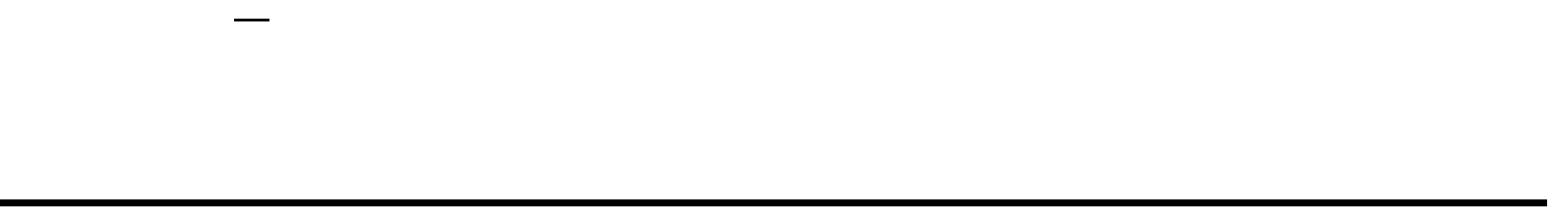

Pol. Con. (Edición núm. 7) Vol. 2, No 6, junio 2017, pp. 1081-1102, ISSN: 2550 - 682X 
Aplicación de la ecuación de continuidad:

Q Teórico $=\mathrm{A}_{1} * \mathrm{~V}_{1}$

Q Teórico $=6,82 * 10^{-4} * 0,0522$

Q Teórico $=35,60 * 10^{-6}\left(\mathrm{~m}^{3} / \mathrm{s}\right)$

Determinación del coeficiente de descarga $\mathrm{Cd}$ :

\section{D) Procedimiento experimental de uso académico para la determinación del coeficiente de}

\section{descarga}

Ver referencia de diseño, construcción y disposición final del tubo de Venturi en las figuras 2, figura 3 y figura 4.

1) Inicialmente se mide en el tubo de Venturi la sección transversal mayor y menor.

2) Se llena con agua el Frasco de Mariotte hasta unos $2 \mathrm{~cm}$ debajo de su tapa superior.

3) Se abre al mismo tiempo la llave de salida de agua en el frasco de Mariotte y la llave de salida en el tubo de Venturi, se quitan las burbujas de aire mediante las válvulas del tubo de Venturi y se comprueba que el flujo sea a tubo lleno y se estabilice. 
4) Una vez estabilizado el caudal se toman datos de volumen (V) y tiempo (T) de llenado de la probeta que se encuentra en la salida de agua en el extremo del dispositivo, los volúmenes de llenado considerados son $100 \mathrm{~cm}^{3}, 200 \mathrm{~cm}^{3}$ y $300 \mathrm{~cm}^{3}$. Para cada volumen de llenado se toman 5 tiempos. En cada momento se debe verificar que el nivel de agua en el frasco de Mariotte nunca se encuentre por debajo del extremo inferior de su tubería.

5) Se verifica mediante el cálculo del número de Reynolds que el flujo se encuentra en régimen laminar.

6) Se repite el procedimiento de los literales 1) y 2)

7) Se mide la diferencia de altura producida en el mercurio del tubo de Venturi, la misma en régimen laminar debe ser constante.

8) Con los datos experimentales obtenidos se determina el coeficiente de descarga.

\section{Conclusiones.}

Al analizar el número de Reynolds para la sección transversal mayor y la sección transversal menor, se determina que el flujo es laminar y es una condición necesaria para la aplicación de la Ecuación de Bernoulli. Ver tabla 1.

El frasco de Mariotte utilizado en el sistema de tubo de Venturi permite obtener un caudal constante, esto se desprende del cálculo de caudal práctico realizado. Ver la tabla 2.

El sistema de tubo de Venturi utilizado cumple con los requerimientos para la aplicación de la ecuación de Bernoulli, que son que el flujo debe encontrarse en régimen laminar y tener un caudal constante. 
Se determinó en laboratorio el caudal práctico promedio, al medir los tiempos de llenado de la probeta que se coloca a la salida del agua del modelo tubo de Venturi.

Para determinar el caudal teórico se utilizó la Ecuación de Continuidad, la Ecuación de Bernoulli y la diferencia de presión entre el la sección transversal mayor y la sección transversal menor, marcada por la diferencia de altura del mercurio del tubo de Venturi utilizado.

Se determina experimentalmente en laboratorio el valor del coeficiente de descarga, al comparar por cociente el valor del caudal práctico con el valor del caudal teórico. Al analizar el valor de coeficiente de descarga $(0,962)$, se desprende que las pérdidas de energía son mínimas

Como resultado de esta investigación se ha determinado un procedimiento experimental de laboratorio para uso académico, que permite realizar una práctica de hidrodinámica para la determinación del coeficiente de descarga en el modelo de tubo de Venturi utilizado.

\section{Recomendaciones.}

Utilizar el sistema de tubo de Venturi utilizado y la referencia de procedimiento experimental, para la enseñanza de la hidrodinámica como lo puede ser para la determinación de las pérdidas de energía.

Elaborar una guía de práctica para la determinación del coeficiente de descarga del modelo de tubo de Venturi utilizado.

De acuerdo al modelo de tubo de Venturi utilizado obtener los recursos necesarios para la construcción de réplicas. 


\section{Agradecimiento.}

Se agradece el apoyo de: la Unidad de Física de la Universidad Central del Ecuador, los compañeros Docentes en esta investigación, los Instructores de Física, los estudiantes de las Facultad de Ciencias Agrícolas, los estudiantes de la Facultad de Ingeniería Ciencias Físicas y Matemática.

\section{Bibliografía.}

Martínez E., Navarrete C. 2000. Diseño e Implementación de un Sistema de Bombeo de Aguas Residuales para una Urbanización. Espol. Ecuador

Serway, R. 2008. Física para Ciencias e Ingeniería. Cengage Learning Editores, S.A. de C.V. México, D.F.

Frish, S. 1977. Curso de Física General. Tomo 3. Editorial Mir. Moscú.

Alonso, M. 1979. Física Mecánica y Termodinámica. Addison - Wesley Iberoamericana. Estados Unidos.

Halliday D., Resnick R., Walker J. 2005. Fundamentos de la Física. Volumen 2 Sexta edición en español CECSA. México.

Sears F., Zemansky M., Young H. 2006. Física Universitaria. Fondo Educativo Iberoamericano, S. A. Wilmintong, Delaware. Estados Unidos de América.

Bueche F. 2006. Física General. Décima edición Mc Graw Hill. México DF.

Hewitt P. 2007. Física Conceptual. Pearson educación. México.

Serway, Raymond A. 2008. Física para Ciencias e Ingeniería. Cengage Learning Editores, S.A. de C.V. México, D.F.

Giancolli D. 2009. Física. Sexta edición, Pearson educación. México.

Arregui De La Cruz F., Cabrera Rochera E., Cobacho Jordán R., Soriano Olivares J., Gómez Sellés E. 2017. Apuntes de mecánica de fluidos. Editorial Universitat Politécnica de Valencia. España.

Giles R. 1967. Mecánica de los fluidos e Hidráulica. Mc Graw-Hill. U.S.A.

Potter M. y Wiggert C. 1998. Mecánica de los fluidos. Prentice Hall. México. 